\title{
62-year-old woman with PET-positive solitary pulmonary nodule
}

\author{
Biplab Saha (1), ' Woon Hean Chong (i) , ${ }^{2}$ Hau Chieng, ${ }^{2}$ Amit Chopra ${ }^{2}$
}

${ }^{1}$ Pulmonary and Critical Care Medicine, Ozarks Medical Center, West Plains, Missouri, USA

${ }^{2}$ Pulmonary and Critical Care Medicine, Albany Medical

Center, Albany, New York, USA

Correspondence to

Dr Biplab Saha;

spanophiliac@yahoo.com

Accepted 7 June 2021

Check for updates

(C) BMJ Publishing Group Limited 2021. No commercial re-use. See rights and permissions. Published by BMJ.

To cite: Saha B, Chong WH, Chieng $\mathrm{H}$, et al. BMJ Case

Rep 2021;14:e243695.

doi:10.1136/bcr-2021-

243695

\section{DESCRIPTION}

A 62-year-old woman was referred to the pulmonary office for an incidentally identified solitary pulmonary nodule. The patient underwent CT of the abdomen and pelvis for evaluation of epigastric abdominal pain and was found to have a pulmonary nodule in the lateral segment of the right middle lobe (RML). Her medical history was significant for hypertension. She reported bronchitis-like symptoms characterised by cough, sputum production, low-grade fever and malaise several months ago, which resolved over a few weeks. At the time of evaluation, she denied any systemic or respiratory symptoms. She was a never smoker and worked as an ophthalmology nurse practitioner. She had no history of tuberculosis (TB) or known exposure to patients with TB. She never travelled outside the USA but lived in a Histoplasma endemic rural midwestern state. She was a never smoker and had no pets at home. Her physical examination was completely normal. A dedicated CT scan of the chest demonstrated a mildly irregular, non-calcified $1.4 \times 1.0 \mathrm{~cm}$ subpleural pulmonary nodule in the RML (figure 1A). There was no hilar or mediastinal lymphadenopathy. A whole-body positron emission tomography (PET) scan revealed a hypermetabolic RML lung nodule with a standard uptake value of 3.6 (figure 1B). There were no hypermetabolic intrathoracic lymph nodes or evidence of extrathoracic disease.

A CT guided core needle biopsy of the nodule was performed. Histopathological analysis showed a parasite with a thick multi-layered cuticular layer measuring $85 \mu \mathrm{m}$ in cross-sectional diameter, lodged in a small pulmonary artery branch (figure 2A). A detailed analysis of the parasitic morphology was limited due to advanced decomposition. There was lymphocytic infiltration of the vessel with disruption of the endothelial layer. Perivascular granulomatous inflammation was also seen. Given the presence of the thick cuticular layer, the parasite was thought to be Dirofilaria immitis. The filaria IgG4 antibody, measured by ELISA, was obtained to assist with diagnostic certainty. The serological assay showed a high antibody level but still within equivocal range (IgG4 antibody 2.7, negative test $<1.5$, positive test $>3$ ). Based on the compatible clinical presentation, the morphology of the degenerated parasite and equivocal serological assay, the patient was diagnosed with human pulmonary dirofilariasis (HPD) and managed conservatively. A repeat CT scan approximately after 1 year revealed a reduction in the size $(1.1 \times 0.9 \mathrm{~cm})$ and the solidity of the nodule (figure $2 \mathrm{~B})$.
HPD is a zoonotic disease caused by the parasite D. immitis, also known as dog heartworm. HPD is a global disease, but the true incidence and prevalence are unknown. ${ }^{1}$ Several reasons contribute to the difficulty in the true estimation of the disease burden. First, many patients with HPD are asymptomatic, and the reported cases used for retrospective analysis may only represent the 'tip of the iceberg'. ${ }^{2}$ Second, not all individuals exposed to $D$. immitis develop a pulmonary nodule or may develop transitionary nodule, leading to a false estimation of true prevalence. ${ }^{3}$ Third, an accurate diagnosis of HPD may be missed unless carefully sought after. ${ }^{45}$ Serological evaluation of a population may provide a more accurate approximation of true prevalence, but large-scale seroepidemiological testing is challenging in the absence of commercially available diagnostic tests. Based on available serological data, the prevalence of HPD is likely higher than previously thought, and the seropositivity rate in humans roughly corresponds to that of canine hosts in an endemic area. ${ }^{16}$ In the USA, the disease appears to be more common in the Southeastern states along the Gulf and Atlantic coasts. ${ }^{7}$ Domestic and wild dogs are the primary definitive hosts, and the female mosquitoes of several species act as a vector. Other definitive hosts include jackals, coyotes and wolves. In definitive hosts, D. immitis causes cardiopulmonary dirofilariasis, which can be a fatal condition. Humans are incidental hosts.

The life cycle of D. immitis is composed of a definite vertebrate host and a vector. The vector deposits the infectious larval form, L3 in the wound of the definitive host during a blood meal. The L3 larval form subsequently moults into L4 and L5 (preadult form) within 50-70 days after infection. The preadult parasites invade the capillaries in the subepidermal layer. Due to the thinness of the membrane, the parasite commonly invades the venous side of the capillary bed to enter the circulation. However, the parasite might also gain access to the vasculature through the arterial side. ${ }^{8}$ The preadult worms are detectable in the right ventricle and pulmonary circulation $70-85$ days after infection. The parasite reaches sexual maturity in approximately 4 months after the inoculation and able to produce microfilariae (L1) about 6-9 months after infection. ${ }^{1}$ The subsequent development of L1 to infectious L3 form occurs in the vector after a blood meal from an infected host. In humans, the development of the preadult parasite into adult worms is arrested. The preadult worms can reach the right ventricle and pulmonary 


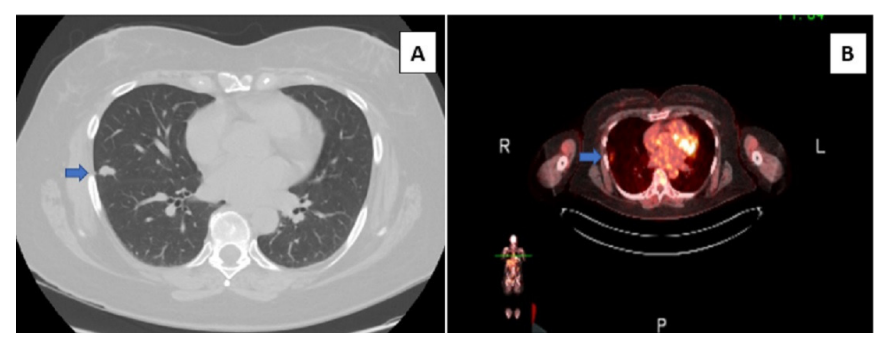

Figure 1 Axial computed tomography (CT) of the chest revealed mildly irregular subpleural pulmonary nodule in the lateral segment of the right middle lobe (A). There was mild pleural thickening (arrow) adjacent to the lesion. B) Positron emission tomography (PET) scan showing hypermetabolic RML (arrow) nodule with a standard uptake value (SUV) of 3.6.

artery but do not develop into sexually mature form capable of producing microfilariae.

HPD typically presents as an incidentally identified pulmonary nodule on imaging and often raises concern for pulmonary malignancy. In a minority of patients (up to 40\%), an acute pneumonitis picture may be seen. ${ }^{9} 10$ These patients become symptomatic during the acute phase of the disease when the worm gets lodged in small pulmonary artery branches causing vascular occlusion and pulmonary infarction. ${ }^{11}$ The mechanical irritation from the parasite and moulting fluid may cause endothelialitis, periarteritis and haemorrhage in the vessel wall. ${ }^{8}$ These causes damage to the vessel wall leading to oozing of intravascular content into the perivascular space and development of interstitial oedema. The interstitial fluid may reach the pleural space causing pleural effusion. ${ }^{13}$ The parasite may also escape to the perivascular space and subsequently lead to the development of granulomatous inflammation. Humans mount an exuberant immunological response to the parasite leading to the eventual destruction of the parasite. Development of pulmonary nodule is the end result of the inflammatory cascade. The acute phase may be characterised by fever, cough, sputum production, chest pain, wheezing, haemoptysis and malaise. Chest radiology typically demonstrates patchy, non-nodular opacity.

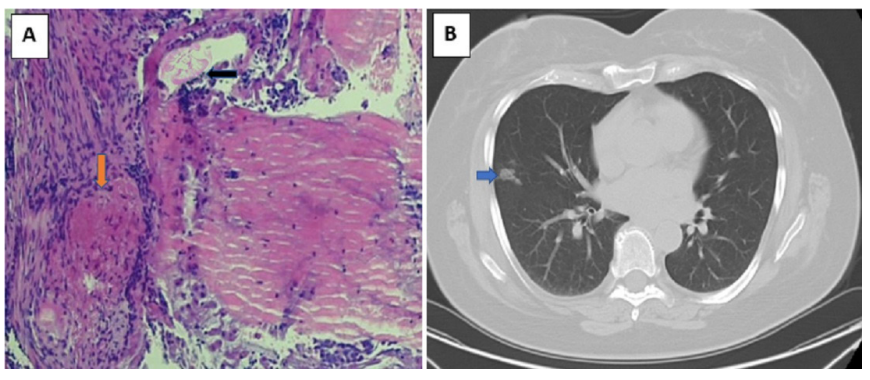

Figure 2 (A) Histopathological analysis of the core needle biopsy of the pulmonary nodule showed a parasite with thick multi-layered cuticle lodged in the small pulmonary artery branch (black arrow). The parasite measured $85 \mu \mathrm{m}$ in cross-sectional diameter and demonstrated advanced stages of decomposition. Degenerating muscular ridges were also seen. The parasitic morphologies were thought to be consistent with Dirofilaria immitis. There is lymphocytic infiltrate of the disrupted vascular endothelium. There are areas of necrosis surrounding the lesion. In addition, perivascular granulomatous inflammation was noted (orange arrow). (B) CT chest obtained approximately after 1 year revealed reduction in the size and solidity of the lesion (blue arrow).
The pulmonary nodules in HPD are predominantly solitary $(75 \%-95 \%) .^{14}$ The lesions are of variable size measuring $0.5-4.5 \mathrm{~cm} .{ }^{10}$ The nodules are more subpleural than central in location and are commonly seen in the right lower lobe (RLL). ${ }^{1115}$ The RLL predominance is thought to be secondary to a larger surface area and higher blood flow to the RLL compared with other lung lobes. The nodules typically have a smooth border with rare reports of perturbation. ${ }^{16}$ Sometimes the nodules can be calcified. ${ }^{7}$ The nodules might persist or disappear over time. The pulmonary nodule in our patient demonstrated hypermetabolic activity on PET scan, a feature that has not been reported in the literature. Three previous cases reported minimal to no 18-fluorodeoxyglucose uptake in the nodule. ${ }^{17-19}$ The PET positivity may also complicate the presurgical differentiation between HPD and malignancy.

The diagnosis of HPD is rarely considered during the initial evaluation of a pulmonary nodule. As a result, HPD is typically diagnosed following histopathological analysis of surgically resected specimen either by video-assisted thoracoscopic surgery or thoracotomy. Fine needle aspirations generally are nondiagnostic; only two other cases except ours have been reported in the literature. ${ }^{20} 21$ The parasite may be difficult to identify on histopathology due to a variable degree of degeneration. $D$. immitis can be seen lodged in a small pulmonary artery branch or in the perivascular space. A thick, multi-layered, smooth cuticular layer may be the only identifiable clue. The presence of thin wall tubules inside the cuticular layer signifies genital and gastrointestinal tracts. ${ }^{22}$ The arteries may show evidence of endothelialitis, lymphocytic infiltration, intimal hyperplasia and vascular disruption.

Serology and molecular testing may play a crucial role in the diagnosis of HPD. The filarial IgG4 antibody test used for our patient is not specific for D. immitis. This ELISA-based test uses a purified antigen from Brugia malayi and can identify exposure to D. immitis, Wuchereria bancrofti, B. malayi and Onchocerca volvulus. Although not available in commercial laboratories, ELISA using Di22 and a recombinant antigen, beta-galactosidase D. immitis recombinant fusion protein, have demonstrated high sensitivity and specificity for the diagnosis of HPD. ${ }^{23} 24$ However, it is crucial to emphasise that the presence of anti- $D$. immitis antibody in the patient's serum indicates prior exposure to the parasite and does not necessarily implicate HPD as the cause of pulmonary nodule. Detection of parasitic DNA by PCR from degenerating parasite on the histopathological specimen may also provide diagnostic certainty. Wolbachia is a symbiotic intracellular bacteria that is crucial for the development of the $D$. immitis parasite. Serological testing for anti-Wolbachia antibody

\section{Learning points}

- Human pulmonary dirofilariasis (HPD) is a rare zoonotic disease that can present as both pneumonitis or pulmonary nodule.

- The pulmonary nodule can be positron emissiontomography positive.

- Identification of the parasite may be challenging due to the variable degree of degeneration. Dirofilaria immitis can be recognised by the presence of its characteristic multi-layered thick cuticle lodged intravascularly or in the perivascular space.

- Prelung resection identification of HPD may allow for conservative therapy and avoidance of invasive surgery. 
or identification of its molecular components on histopathological samples may also provide diagnostic clues. If a diagnosis of HPD can be made before surgical resection, no specific treatment is required.

Contributors $\mathrm{BS}, \mathrm{WHC}, \mathrm{HC}$ and $\mathrm{AC}$ were involved in the planning, collection of data, preparation of the initial and final manuscript.

Funding The authors have not declared a specific grant for this research from any funding agency in the public, commercial or not-for-profit sectors.

Competing interests None declared.

Patient consent for publication Obtained.

Provenance and peer review Not commissioned; externally peer reviewed.

ORCID iDs

Biplab Saha http://orcid.org/0000-0002-3595-8804

Woon Hean Chong http://orcid.org/0000-0002-2070-3585

\section{REFERENCES}

1 Simón F, Siles-Lucas M, Morchón R, et al. Human and animal dirofilariasis: the emergence of a zoonotic mosaic. Clin Microbiol Rev 2012;25:507-44.

2 Simón F, López-Belmonte J, Marcos-Atxutegi C, et al. What is happening outside North America regarding human dirofilariasis? Vet Parasitol 2005;133:181-9.

3 Cordero M, Muro A, Simón F, et al. Are transient pulmonary solitary nodules a common event in human dirofilariosis? Clin Investig 1992:70:437-40.

4 Harrison EG, Thompson JH. Dirofilariasis of human lung. Am J Clin Pathol 1965:43:224-34.

5 Green LK, Ansari MQ, Schwartz MR, et al. Non-Specific fluorescent whitener stains in the rapid recognition of pulmonary dirofilariasis: a report of 20 cases. Thorax 1994;49:590-3.

6 Espinoza E, Cordero M, Muro A, et al. Anti-Dirofilaria immitis IgE: seroepidemiology and seasonal variation in an exposed human population. Trop Med Parasitol 1993:44:172-6

7 Ciferri F. Human pulmonary dirofilariasis in the United States: a critical review. Am J Trop Med Hyg 1982:31:302-8.
8 Theis JH. Public health aspects of dirofilariasis in the United States. Vet Parasitol 2005:133:157-80.

9 Ro JY, Tsakalakis PJ, White VA, et al. Pulmonary dirofilariasis: the great imitator of primary or metastatic lung tumor. A clinicopathologic analysis of seven cases and a review of the literature. Hum Pathol 1989;20:69-76.

10 Flieder DB, Moran CA. Pulmonary dirofilariasis: a clinicopathologic study of 41 lesions in 39 patients. Hum Pathol 1999;30:251-6.

11 Atsumi $\mathrm{E}$, Matsumoto $\mathrm{H}$, Taira $\mathrm{N}$, et al. Thirteen cases of pulmonary dirofilariasis in a single institution in Okinawa island. Virchows Arch 2019;475:335-40.

12 Araya J, Kawabata Y, Tomichi $\mathrm{N}$, et al. Allergic inflammatory reaction is involved in necrosis of human pulmonary dirofilariasis. Histopathology 2007:51:484-90.

13 Faruqi MA, Ashraf O, Virani A, et al. Rare occurrence: pulmonary dirofilariasis as etiology of eosinophilic pleural effusion. Chest 2020;158:A1250.

14 Selvachandran A, Foley RJ. Subcutaneous and pulmonary dirofilariasis with evidence of splenic involvement. Case Rep Pulmonol 2016;2016:1-4.

15 Milanez de Campos JR, Barbas CS, Filomeno LT, et al. Human pulmonary dirofilariasis: analysis of 24 cases from São Paulo, Brazil. Chest 1997;112:729-33.

16 Solaini L, Gourgiotis S, Salemis NS, et al. A case of human pulmonary dirofilariasis. Int $J$ Infect Dis 2008:12:e147-8.

17 Stone M, Dalal I, Stone C, et al. 18-FDG uptake in pulmonary dirofilariasis. J Radiol Case Rep 2015:9:28-33.

18 Kang HJ, Park YS, Lee C-H, et al. A case of human pulmonary dirofilariasis in a 48-year-old Korean man. Korean J Parasitol 2013:51:569-72.

19 Grapatsas K, Kayser G, Passlick B, et al. Pulmonary coin lesion mimicking lung cancer reveals an unexpected finding: Dirofilaria immitis. J Thorac Dis 2018:10:3879-82.

20 Hawkins AG, Hsiu JG, Smith RM, et al. Pulmonary dirofilariasis diagnosed by fine needle aspiration biopsy. A case report. Acta Cytol 1985;29:19-22.

21 Kelly WT, Firouz-Abadi AA, Roszkowski A, et al. Pulmonary dirofilariasis diagnosed by computerised tomography scan controlled percutaneous needle aspiration. Aust N Z J Med 1985;15:656-7.

22 Orihel TC, Eberhard ML. Zoonotic filariasis. Clin Microbiol Rev 1998;11:366-81.

23 Perera L, Muro A, Cordero M, et al. Evaluation of a $22 \mathrm{kDa}$ Dirofilaria immitis antigen for the immunodiagnosis of human pulmonary dirofilariosis. Trop Med Parasitol 1994:45:249-52.

24 Sun S, Sugane K. Immunodiagnosis of human dirofilariasis by enzyme-linked immunosorbent assay using recombinant DNA-derived fusion protein. J Helminthol 1992;66:220-6.

Copyright 2021 BMJ Publishing Group. All rights reserved. For permission to reuse any of this content visit https://www.bmj.com/company/products-services/rights-and-licensing/permissions/

BMJ Case Report Fellows may re-use this article for personal use and teaching without any further permission.

Become a Fellow of BMJ Case Reports today and you can:

- Submit as many cases as you like

Enjoy fast sympathetic peer review and rapid publication of accepted articles

- Access all the published articles

Re-use any of the published material for personal use and teaching without further permission

Customer Service

If you have any further queries about your subscription, please contact our customer services team on +44 (0) 2071111105 or via email at support@bmj.com.

Visit casereports.bmj.com for more articles like this and to become a Fellow 